\title{
Modeling-Error Based Adaptive Fuzzy Sliding Mode Control for Trajectory-Tracking of Nonlinear Systems
}

\author{
Shuanghe $\mathrm{Yu}^{*}$, Xinghuo $\mathrm{Yu}^{* *}$, Mehmet Önder Efe ${ }^{* * *}$ \\ ${ }^{*}$ Faculty of Informatics and Communication, Central Queensland University, Rockhampton, QLD 4702, Australia. Email: $\underline{\text { s.yu@ecqu.edu.au }}$ \\ ${ }^{* *}$ Faculty of Engineering, Royal Melbourne Institute of Technology, Melbourne, VIC 3001, Australia. E-mail: Email: x.yu@rmit.edu.au \\ **** Department of Mechatronics Engineering, Atilim University, Incek, 06836, Ankara, Turkey. E-mail: onderefe@ieee.org
}

\begin{abstract}
A novel adaptive fuzzy sliding mode control design is developed for trajectory tracking of a class of nonlinear systems in this paper. This control design uses the modelling error to adaptively estimate the deterministic uncertainties as well as the control gain based on the fuzzy systems approach. By this design, the bounds of the uncertainties are not required to be known in advance, and the robust stability of closed loop systems is analysed in the Lyapunov sense. Simulation results are given to demonstrate the improved performance.
\end{abstract}

\section{INTRODUCTION}

The control of nonlinear systems has been an important research topic and many approaches have been proposed (see $[1,2]$ for summary of the state of the art). Feedback linearisation based adaptive control is suitable for the control of nonlinear systems with accurate nominal models or linearly parametrizable dynamical models. However, due to modelling errors, these controls may not be very effective without proper compensation to overcome the modeling error effects. The design of robust control for uncertain nonlinear systems is still a challenging problem.

Sliding mode control (SMC) is well known for handling matched uncertainties ${ }^{[2,3,4]}$. A sliding-mode control law is formulated using a Lyapunov approach to guarantee that the system state first reaches the prescribed sliding mode in finite time from any initial state, and then remains on it thereafter by a discontinuous control. However, SMC suffers from a well known problem - chattering due to the high gain and high-speed switching control. The undesirable chattering may excite previously unmodelled system dynamics and damage actuators, resulting in unpredictable instability. Smoothing techniques such as the boundary layer approach have been employed to reduce its effects at the cost of giving concessions from performance. Therefore, a compromise must be sought between the desired control accuracy and controller bandwidth.

As a model free design method, fuzzy systems have been successfully applied to control complex or ill-defined processes whose mathematical models are difficult to obtain $^{[5]}$. The ability of converting linguistic descriptions into automatic control strategy makes it a practical and

0-7803-7906-3/03/\$17.00 @2003 IEEE. promising alternative to the classical control scheme for achieving control of complex nonlinear systems. A major drawback of fuzzy control systems is that the fuzzy rules must be previously tuned by time-consuming trial-and-error procedures because of lack of adequate analysis and design techniques. To overcome this problem, some research has been focused on the Lyapunov synthesis approach to construct stable adaptive fuzzy controllers ${ }^{[5-9]}$. The basic idea of most of these works is that with the universal approximation ability of fuzzy systems, the system uncertainties can be represented by linearly parameterized uncertainties so that the standard parametric adaptive techniques can be utilized.

Similar to the conventional adaptive control, adaptive fuzzy control can be categorized into "direct", "indirect" and "composite" schemes, respectively, according to the type of fuzzy rules ${ }^{[5]}$. For all the three methods, the adjustable fuzzy parameters are updated on-line by the tracking-error based adaptive laws for achieving specified tracking performance. Unfortunately, it is not sufficient to have successful function approximation by using tracking error only, although the convergence of fuzzy approximator may not be of interest ${ }^{[10]}$. However, accurate function approximation can facilitate achieving the tracking error specifications because tracking error is a direct result of the function approximation error ${ }^{[11]}$. Recently, modified "direct" and "indirect" approaches have been proposed according to the type of error the adaptive laws are based on, and a hybrid adaptive fuzzy control scheme based on the tracking error and modelling error has been developed for achieving improved tracking ability and parameter convergence ${ }^{[12]}$. However this kind of hybrid methods may lead to an undesirable adaptive approximation with perfect modelling but nonzero tracking error, resulting in unsatisfactory transient performance of the closed loop system.

The apparent similarities between sliding mode control and fuzzy control motivate considerable research efforts in combining the two approaches for achieving more superior performances such as overcoming some limitations of the traditional sliding mode contro ${ }^{[13-23]}$. The approaches tend to fall in two different classes of algorithms: non-adaptive and adaptive. The first approach attempts to replace the linear 
control in conventional boundary layer with an equivalent fuzzy control which may be nonlinear for the same purpose of alleviating the chattering ${ }^{[13-16]}$. However, the advantages of these treatments are not clear compared with conventional boundary method because the control performance is influenced by the width, not the form of boundary layer ${ }^{[2]}$. The second approach is similar to the popular adaptive fuzzy control but is augmented with a sliding mode control scheme to deal with the residual modelling error ${ }^{[17-23]}$, so that and the limitation on the known bounds of uncertainties can be released by an on-line estimation using fuzzy systems ${ }^{[19-23]}$. Although the trajectory-tracking objective can be guaranteed, these tracking-error based adaptive fuzzy sliding mode control schemes also cannot ensure a successful function approximation.

In this paper, a new adaptive fuzzy sliding mode controller for uncertain nonlinear systems is proposed. The modellingerror based adaptive laws are used to adjust three separate fuzzy systems for accurately approximating the unknown deterministic nonlinear uncertainties as well as the control gain, which are then used in the sliding mode control. As a result, chattering can be effectively reduced to a minimum level.

The remainder of this paper is organized as follows. A statement about the problem concerned and some preliminaries are given in Section II. The main results are presented in Section III, which includes the design of the control structure and adaptation laws with stability and convergence analysis using the Lyapunov approach. A simulation is presented to validate the proposed approach in Section IV. Finally, some conclusions are given in section $\mathrm{V}$.

\section{PROBLEM STATEMENT AND PRELIMINARIES}

A. Problem Statement

Consider the SISO (single-input and single-output) affine nonlinear dynamical system

$$
\left\{\begin{array}{l}
\dot{x}_{1}=x_{2} \\
\dot{x}_{2}=x_{3} \\
\vdots \\
\dot{x}_{n}=f(\mathbf{x})+g(\mathbf{x}) u \\
y=x_{1}
\end{array}\right.
$$

or equivalently

$$
\left\{\begin{array}{l}
x^{(n)}=f(\mathbf{x})+g(\mathbf{x}) u \\
y=x
\end{array}\right.
$$

where $\mathbf{x}=\left[x_{1}, x_{2}, \cdots, x_{n}\right]^{T}=\left[x, \dot{x}, \cdots, x^{(n-1)}\right]^{T} \in \mathbf{\Omega}_{1} \subset R^{n}$ is the system state vector which is assumed to be available for measurement, the scalar $x$ is the variable of interest (for instance the position of mechanical system), $x^{(i)}$ being the $i$ th order time derivative of $x, u(t) \in \Omega_{2} \subset R$ is the control input (for example, the motor torque), $f, g: R^{n} \rightarrow R$ are nonlinear system functions representing the dynamic system behaviour and control gain respectively. Note more general classes of nonlinear dynamic systems can be transformed into this structure ${ }^{[1,2]}$.

In real world applications, the system functions $f(\mathbf{x})$ and $g(\mathbf{x})$ may not be exactly known, which can be split into two parts as

$$
f(\mathbf{x})=\bar{f}(\mathbf{x})+\Delta f(\mathbf{x}), \quad g(\mathbf{x})=\bar{g}(\mathbf{x})+\Delta g(\mathbf{x})
$$

where $\bar{f}(\mathbf{x})$ and $\bar{g}(\mathbf{x})$ denote the nominal parts, and $\Delta f(\mathbf{x})$ and $\Delta g(\mathbf{x})$ the uncertain parts of $f(\mathbf{x})$ and $g(\mathbf{x})$. Here we assume that $\Delta f(\mathbf{x})$ and $\Delta g(\mathbf{x})$ are bounded as

$$
|\Delta f(\mathbf{x})| \leq F(\mathbf{x}),|\Delta g(\mathbf{x})| \leq G(\mathbf{x}), \quad \forall \mathbf{x} \in \mathbf{\Omega}_{1}
$$

where the bound functions $F(\mathbf{x})$ and $G(\mathbf{x})$ can be unknown but non-negative. Without loss of generality, we have the following assumption:

Assumption 1: The gain $g(\mathbf{x})$ is strictly positive and globally bounded away from zero by a known constant $g_{l}$, i.e.,

$$
g(\mathbf{x}) \geq g_{l}>0 \quad \text { for all } \mathbf{x} \in \mathbf{\Omega}_{1}
$$

Note that $g(\mathbf{x})$ can be assumed negative, and the controller can be similarly derived.

In the following, $\quad \mathbf{x}_{d}=\left[x_{d}, \dot{x}_{d}, \cdots, x_{d}^{(n-1)}\right]^{T} \in \mathbf{\Omega}_{3} \subset R^{n}$ represents a given desired state trajectory which is assumed to be bounded and continuously differentiable. Define $e=x_{d}-x$ as the tracking error and the tracking error vector as

$$
\mathbf{e}=\mathbf{x}_{d}-\mathbf{x}=\left[e, \dot{e}, \cdots, e^{(n-1)}\right]^{T}
$$

The control objective is to determine a robust feedback control $u\left(\mathbf{x}, \mathbf{x}_{d}\right)$ to drive the state $\mathbf{x}$ to asymptotically track a desired reference signal $\mathbf{x}$ in the presence of model imprecision on $f(\mathbf{x})$ and $g(\mathbf{x})$, i.e., the tracking error $\mathbf{e} \rightarrow 0$, as $t \rightarrow \infty$, under the condition that all involved signals in the closed loop remain bounded.

If the bound functions $F(\mathbf{x})$ and $G(\mathbf{x})$ are known, the traditional sliding mode control with high switching gain can guarantee the robust tracking. However, such a control approach entails unnecessarily large control energy and may result in severe chattering. Therefore, this article pursues a new kind of active control approach, which attempts to approximate the unknown functions $\Delta f(\mathbf{x})$ and $\Delta g(\mathbf{x})$ in order to minimize the modeling errors. A direct benefit from it is the switching gain can be reduced to a minimum level.

B. Sliding Mode Control

For system (1), define a sliding surface in the space of the tracking-error vector by a scalar equation:

$$
s=e^{(n-1)}+c_{n-1} e^{(n-2)}+\cdots+c_{1} e=0
$$

The coefficients $c_{i}, i=1,2, \cdots, n-1$ are positive constants such that the polynomial $\lambda^{(n-1)}+c_{n-1} \lambda^{(n-2)}+\cdots+c_{1}$ is Hurwitz. Now the $n$ th-order trajectory-tracking problem in $\mathbf{x}$ becomes 
a first-order stabilization problem in $s$ : designing a control law $u$ such that the state trajectory is forced onto the sliding surface $s(\mathbf{x}, t)=0$ in finite time and then remain sliding on it. Define a Lyapunov function as

$$
V=\frac{1}{2} s^{2}
$$

A sufficient condition for the stability of the system is

$$
\dot{V}=\frac{1}{2} \frac{d t}{d} s^{2} \leq-\eta|s|, \quad \eta>0
$$

which leads to the reaching condition for $s(\mathbf{x}, t)=0$ as

$$
s \dot{s} \leq-\eta|s|
$$

Differentiating $s$ along the system trajectories (1), we have

$$
\dot{s}=\sum_{i=1}^{n-1} c_{i} e^{(i)}+x_{d}^{(n)}-f(\mathbf{x})-g(\mathbf{x}) u
$$

If the plant dynamics is known, i.e., the functions $f$ and $g$ are completely known, the desired sliding mode control law can be chosen as

$$
u^{d}=u_{e q}^{d}+u_{s w}^{d}=\frac{1}{g(\mathbf{x})}\left(\sum_{i=1}^{n-1} c_{i} e^{(i)}-f(\mathbf{x})+x_{d}^{(n)}+k \operatorname{sign}(s)\right),
$$$$
k \geq \eta
$$

where $u_{e q}^{d}$ is the ideal equivalent control part which can achieve the sliding condition $\dot{s}=0$ when the system is on the sliding surface $s=0$, and $u_{s w}^{d}$ is the ideal switching control which can achieve the reaching condition (10) together with $u_{e q}^{d}$.

However, $f(\mathbf{x})$ and $g(\mathbf{x})$ are actually unknown, so we cannot use them to construct the equivalent control in (12). Instead, we can only use the nominal portions $\bar{f}(\mathbf{x})$ and $\bar{g}(\mathbf{x})$ to replace $f(\mathbf{x})$ and $g(\mathbf{x})$. In order to satisfy the reaching condition (10), the system uncertainties have to be compensated by a switching control. The resulting sliding mode controller is

$$
\begin{aligned}
u & =u_{e q}+u_{s w} \\
& =\frac{1}{\bar{g}(\mathbf{x})}\left(\begin{array}{c}
\sum_{i=1}^{n-1} c_{i} e^{(i)}-\bar{f}(\mathbf{x})+x_{d}^{(n)} \\
+\frac{\bar{g}(\mathbf{x})}{g_{l}}\left(F(\mathbf{x})+G(\mathbf{x})\left|u_{e q}\right|+\eta\right) \operatorname{sign}(s)
\end{array}\right)
\end{aligned}
$$

which can still guarantee the reaching condition (10). However, the large uncertainty bounds in the switching control of (13) usually result in large and chattering control efforts, which are usually undesirable in most applications.

\section{Fuzzy Logic Systems}

The basic configuration of a fuzzy logic system consists of a fuzzifier, a fuzzy rule base, a fuzzy inference engine and a defuzzifier. The fuzzy inference engine uses the fuzzy IFTHEN rules to perform a mapping from an input vector $\mathbf{x}=\left[x_{1}, x_{2}, \cdots, x_{n}\right]^{T}$ to an output scalar $y$. The fuzzy rule base consists of a collection of fuzzy IF-THEN rules in the following form
$R^{j}$ : If $x_{1}$ is $F_{1}^{j}$ and $\ldots$ and $x_{n}$ is $F_{n}^{j}$, then $y$ is $\theta_{j}$

where $F_{i}{ }^{j}, i=1, \cdots, n$ are the fuzzy variables characterized by membership functions $\mu_{F_{j}^{\prime}}\left(x_{i}\right)$ and $\theta_{j}$ is the corresponding value of the output fuzzy singleton. The output of the fuzzy system with singleton fuzzification, product inference and centre average defuzzification can be expressed as

$$
y=\frac{\sum_{j=1}^{M} \theta_{j}\left(\prod_{i=1}^{n} \mu_{F_{i}^{\prime}}\left(x_{i}\right)\right)}{\sum_{j=1}^{M}\left(\prod_{i=1}^{n} \mu_{F_{i}^{j}}\left(x_{i}\right)\right)}=\boldsymbol{\theta}^{T} \xi(\mathbf{x})
$$

where $M$ is the total number of the fuzzy rules, $\boldsymbol{\theta}=\left[\theta_{1}, \theta_{2}, \cdots, \theta_{M}\right]^{T}$ is the adjustable parameter vector, $\boldsymbol{\xi}(\mathbf{x})=\left[\xi_{1}(\mathbf{x}), \xi_{2}(\mathbf{x}), \cdots, \xi_{M}(\mathbf{x})\right]^{T}$ is the vector of the fuzzy basis functions $\xi_{j}(\mathbf{x})=\left(\prod_{i=1}^{n} \mu_{F_{i}^{j}}\left(x_{i}\right)\right) /\left(\sum_{j=1}^{M}\left(\prod_{i=1}^{n} \mu_{F_{i}^{j}}\left(x_{i}\right)\right)\right)$.

Based on the universal approximation theorem [5], the above fuzzy logic system is capable of uniformly approximating any well-defined nonlinear function over a compact set $U$ to any degree of accuracy.

\section{MAIN RESULTS}

\section{A. Fuzzy Sliding-Mode Control}

Due to the universal approximation property, we use two fuzzy logic systems to approximate the unknown nonlinear functions $\Delta f(\mathbf{x})$ and $\Delta g(\mathbf{x})$ respectively:

$$
\Delta \hat{f}\left(\mathbf{x} \mid \boldsymbol{\theta}_{f}\right)=\boldsymbol{\theta}_{f}^{T} \boldsymbol{\xi}(\mathbf{x}), \Delta \hat{g}\left(\mathbf{x} \mid \boldsymbol{\theta}_{g}\right)=\boldsymbol{\theta}_{g}^{T} \boldsymbol{\xi}(\mathbf{x})
$$

where $\boldsymbol{\theta}_{f}$ and $\boldsymbol{\theta}_{g}$ are adjustable parameter vectors. The fuzzy approximators are valid under the following assumptions

Assumption 2: Let $\mathbf{x}$ belong to a compact set $U_{x}=\left\{x \in R^{n}:\|x\| \leq m_{x}<\infty\right\}$. It is assumed that the optimal vectors $\boldsymbol{\theta}_{f}^{*}$ and $\boldsymbol{\theta}_{g}^{*}$ that minimize the modelling errors lie in some convex regions

$$
\begin{gathered}
M_{\boldsymbol{\theta}_{f}}=\left\{\boldsymbol{\theta}_{f}=\left[\theta_{f}^{1}, \theta_{f}^{2}, \cdots, \theta_{f}^{M}\right]^{T} \in R^{M}:\left\|\boldsymbol{\theta}_{f}\right\| \leq m_{\boldsymbol{\theta}_{f}}\right\} \\
M_{\boldsymbol{\theta}_{g}}=\left\{\begin{array}{r}
\boldsymbol{\theta}_{g}=\left[\theta_{g}^{1}, \theta_{g}^{2}, \cdots, \theta_{g}^{M}\right]^{T} \in R^{M}:\left\|\boldsymbol{\theta}_{g}\right\| \leq m_{\boldsymbol{\theta}_{g}} ; \\
\theta_{g}^{l} \geq \delta>0, l=1,2, \cdots, M
\end{array}\right\} \\
\boldsymbol{\theta}_{f}^{*}=\arg \min _{\boldsymbol{\theta}_{f} \in M_{\mathbf{\theta}_{f}}}\left[\sup _{\mathbf{x} \in U_{x}}\left|\Delta \hat{f}\left(\mathbf{x} \mid \boldsymbol{\theta}_{f}\right)-\Delta f(\mathbf{x})\right|\right] \\
\boldsymbol{\theta}_{g}^{*}=\arg \min _{\boldsymbol{\theta}_{g} \in M_{\mathbf{\theta}_{g}}}\left[\sup _{\mathbf{x} \in U_{x}}\left|\Delta \hat{g}\left(\mathbf{x} \mid \boldsymbol{\theta}_{g}\right)-\Delta g(\mathbf{x})\right|\right]
\end{gathered}
$$

where the radii $m_{x}, m_{\boldsymbol{\theta}_{f}}$ and $m_{\boldsymbol{\theta}_{g}}$ are positive constants.

Therefore, the unknown nonlinear functions $\Delta f(\mathbf{x})$ and $\Delta g(\mathbf{x})$ can be expressed as 


$$
\begin{aligned}
& \Delta f(\mathbf{x})=\Delta \hat{f}\left(\mathbf{x} \mid \boldsymbol{\theta}_{f}^{*}\right)+\omega_{f}(\mathbf{x})=\boldsymbol{\theta}_{f}^{* T} \boldsymbol{\xi}(\mathbf{x})+\omega_{f}(\mathbf{x}) \\
& \Delta g(\mathbf{x})=\Delta \hat{g}\left(\mathbf{x} \mid \boldsymbol{\theta}_{g}^{*}\right)+\omega_{g}(\mathbf{x})=\boldsymbol{\theta}_{g}^{* T} \xi(\mathbf{x})+\omega_{g}(\mathbf{x})
\end{aligned}
$$

where $\omega_{f}, \omega_{g}$ represent the modelling errors and $\left|\omega_{f}(x)\right| \leq \bar{\omega}_{f}$ and $\left|\omega_{g}(x)\right| \leq \bar{\omega}_{g}$ with $\bar{\omega}_{f}$ and $\bar{\omega}_{g}$ being the positive upper bounds.

Assume the estimations $\Delta \hat{f}\left(\mathbf{x} \mid \boldsymbol{\theta}_{f}\right)$ and $\Delta \hat{g}\left(\mathbf{x} \mid \boldsymbol{\theta}_{g}\right)$ are ideal, we can then redesign the sliding mode controller (13) as

$$
\begin{aligned}
\hat{u} & =\hat{u}_{e q}+\hat{u}_{s w} \\
= & \frac{1}{\bar{g}(\mathbf{x})+\Delta \hat{g}\left(\mathbf{x} \mid \boldsymbol{\theta}_{g}\right)}\left(\begin{array}{r}
\sum_{i=1}^{n-1} c_{i} e^{(i)}-\bar{f}(\mathbf{x})-\Delta \hat{f}\left(\mathbf{x} \mid \boldsymbol{\theta}_{f}\right) \\
+x_{d}^{(n)}+\hat{k}\left(\mathbf{x} \mid \boldsymbol{\theta}_{k}\right) \operatorname{sign}(s)
\end{array}\right)
\end{aligned}
$$

where $\hat{k}\left(\mathbf{x} \mid \boldsymbol{\theta}_{k}\right)$ is the third fuzzy logic system which is used to estimate the bound of switching gain. Here we call the controller (20) as a fuzzy sliding mode control. Because of the limited approximation ability of fuzzy system, the best fuzzy sliding mode control we can acquire is

$$
\begin{aligned}
\hat{u}^{*} & =\hat{u}_{e q}^{*}+\hat{u}_{s w}^{*} \\
& =\frac{1}{\bar{g}(\mathbf{x})+\hat{g}\left(\mathbf{x} \mid \boldsymbol{\theta}_{g}^{*}\right)}\left(\begin{array}{c}
\sum_{i=1}^{n-1} c_{i} e^{(i)}-\bar{f}(\mathbf{x})- \\
\Delta \hat{f}\left(\mathbf{x} \mid \boldsymbol{\theta}_{f}^{*}\right)+x_{d}^{n}+\hat{k}\left(\mathbf{x} \mid \boldsymbol{\theta}_{k}^{*}\right) \operatorname{sign}(s)
\end{array}\right)
\end{aligned}
$$

where $\hat{k}\left(\mathbf{x} \mid \boldsymbol{\theta}_{k}^{*}\right)=g_{l}^{-1}\left(\bar{g}(\mathbf{x})+\hat{g}\left(\mathbf{x} \mid \boldsymbol{\theta}_{g}^{*}\right)\right)\left(\bar{\omega}_{f}+\bar{\omega}_{g}\left|\hat{u}_{e q}^{*}\right|+\eta\right)$. The controller (21) is called the ideal fuzzy sliding mode control. Compared with the traditional sliding mode control (13), the switching gain in control (21) is reduced greatly without loss of the reaching and sliding condition because of use of approximation of uncertainties. However, the ideal control parameters are not known, so the controller (20) should be equipped with appropriate on-line adaptive algorithms to obtain the ideal controller (21).

\section{B. Function Approximation}

In this section, we develop the adaptive laws for adjusting the parameters of the fuzzy sliding mode controller (20). The goal of parameter adjustment is to make $\boldsymbol{\theta}_{f} \rightarrow \boldsymbol{\theta}_{f}^{*}$ and $\boldsymbol{\theta}_{g} \rightarrow \boldsymbol{\theta}_{g}^{*}$, and consequently make $\Delta \hat{f}\left(\mathbf{x} \mid \boldsymbol{\theta}_{f}\right)$ and $\Delta \hat{g}\left(\mathbf{x} \mid \boldsymbol{\theta}_{g}\right)$ to approximate $\Delta f(\mathbf{x})$ and $\Delta g(\mathbf{x})$ using the modelling errors respectively, therefore $\hat{u} \rightarrow \hat{u}^{*}$.

If the controller (20) is applied into (11), we have

$$
\dot{s}=\Delta \hat{f}\left(\mathbf{x} \mid \boldsymbol{\theta}_{f}\right)-\Delta f(\mathbf{x})+\left(\Delta \hat{g}\left(\mathbf{x} \mid \boldsymbol{\theta}_{g}\right)-\Delta g(\mathbf{x})\right) \hat{u}-\hat{k}\left(\mathbf{x} \mid \boldsymbol{\theta}_{k}\right) \operatorname{sign}(s)
$$

From the viewpoint of system identification, the system (1) is modelled as

$$
\begin{aligned}
\dot{x}_{n}=\bar{f}(\mathbf{x})+\Delta \hat{f}\left(\mathbf{x} \mid \boldsymbol{\theta}_{f}\right)+\left(\bar{g}(\mathbf{x})+\Delta \hat{g}\left(\mathbf{x} \mid \boldsymbol{\theta}_{g}\right)\right) \hat{u}+ \\
\Delta f(\mathbf{x})-\Delta \hat{f}\left(\mathbf{x} \mid \boldsymbol{\theta}_{f}\right)+\left(\Delta g(\mathbf{x})-\Delta \hat{g}\left(\mathbf{x} \mid \boldsymbol{\theta}_{g}\right)\right) \hat{u}
\end{aligned}
$$

then we can define the modelling error as

$$
\begin{aligned}
\omega & =\Delta \hat{f}\left(\mathbf{x} \mid \boldsymbol{\theta}_{f}\right)-\Delta f(\mathbf{x})+\left(\Delta \hat{g}\left(\mathbf{x} \mid \boldsymbol{\theta}_{g}\right)-\Delta g(\mathbf{x})\right) \hat{u} \\
& =\omega_{f}(\mathbf{x})+\widetilde{\boldsymbol{\theta}}_{f}^{T} \boldsymbol{\xi}(\mathbf{x})+\left(\omega_{g}(\mathbf{x})+\widetilde{\boldsymbol{\theta}}_{g}^{T} \boldsymbol{\xi}(\mathbf{x})\right) \hat{u} \\
& =\widetilde{\boldsymbol{\theta}}_{f}^{T} \boldsymbol{\xi}(\mathbf{x})+\widetilde{\boldsymbol{\theta}}_{g}^{T} \boldsymbol{\xi}(\mathbf{x}) \hat{u}+\omega_{f}(\mathbf{x})+\omega_{g}(\mathbf{x}) \hat{u} \\
& =\widetilde{\boldsymbol{\theta}}_{f}^{T} \boldsymbol{\xi}(\mathbf{x})+\widetilde{\boldsymbol{\theta}}_{g}^{T} \boldsymbol{\xi}(\mathbf{x}) \hat{u}+m
\end{aligned}
$$

where $\tilde{\boldsymbol{\theta}}_{f}=\boldsymbol{\theta}_{f}-\boldsymbol{\theta}_{f}^{*}$ and $\tilde{\boldsymbol{\theta}}_{g}=\boldsymbol{\theta}_{g}-\boldsymbol{\theta}_{g}^{*}$ are parameter errors, $\omega$ is the modelling error and $m=\omega_{f}(\mathbf{x})+\omega_{g}(\mathbf{x}) \hat{u}$ is the modelling error residual.

Therefore, the expression (22) can be redefined as

$$
\dot{s}=\omega-\hat{k}\left(\mathbf{x} \mid \boldsymbol{\theta}_{k}\right) \operatorname{sign}(s)=\omega-\left(\boldsymbol{\theta}_{k}^{T} \boldsymbol{\xi}(\mathbf{x})\right) \operatorname{sign}(s)
$$

It is clear that the switching gain $\hat{k}\left(\mathbf{x} \mid \boldsymbol{\theta}_{k}\right)$ is just used to overcome the modelling error $\omega$. Note that when $\omega$ is very small, $\hat{k}\left(\mathbf{x} \mid \boldsymbol{\theta}_{k}\right)$ can be as low as the minimum level $\eta$, and the sliding mode $s=0$ can be still reached in finite time.

In order to obtain the ideal function approximations, the following modelling-error based adaptive laws are proposed to update the parameter vectors of fuzzy systems $\Delta \hat{f}\left(\mathbf{x} \mid \boldsymbol{\theta}_{f}\right)$ and $\Delta \hat{g}\left(\mathbf{x} \mid \boldsymbol{\theta}_{g}\right)$ based on the fact that the optimal parameters are defined with respect to the plant model, not to the tracking performance:

$$
\dot{\boldsymbol{\theta}}_{f}=-\gamma_{1} \omega \xi(\mathbf{x}), \dot{\boldsymbol{\theta}}_{g}=-\gamma_{2} \omega \xi(\mathbf{x}) \hat{u}
$$

where $\gamma_{1}, \gamma_{2}>0$.

If we choose the Lyapunov function as

$$
V=\frac{1}{2 \gamma_{1}} \widetilde{\boldsymbol{\theta}}_{f}^{T} \widetilde{\boldsymbol{\theta}}_{f}+\frac{1}{2 \gamma_{2}} \widetilde{\boldsymbol{\theta}}_{g}^{T} \widetilde{\boldsymbol{\theta}}_{g}
$$

we can easily get

$$
\begin{aligned}
\dot{V} & =\frac{1}{\gamma_{1}} \widetilde{\boldsymbol{\theta}}_{f}^{T} \dot{\boldsymbol{\theta}}_{f}+\frac{1}{\gamma_{2}} \widetilde{\boldsymbol{\theta}}_{g}^{T} \dot{\boldsymbol{\theta}}_{g}=-\omega \widetilde{\boldsymbol{\theta}}_{f}^{T} \boldsymbol{\xi}(\mathbf{x})-\omega \widetilde{\boldsymbol{\theta}}_{g}^{T} \boldsymbol{\xi}(\mathbf{x}) \hat{u} \\
& =-\omega\left(\widetilde{\boldsymbol{\theta}}_{f}^{T} \boldsymbol{\xi}(\mathbf{x})+\widetilde{\boldsymbol{\theta}}_{g}^{T} \boldsymbol{\xi}(\mathbf{x}) \hat{u}\right)=-\omega(\omega-m) \\
& =-\omega^{2}+\omega m \leq-\omega^{2}+|\omega||m|=-|\omega|(|\omega|-|m|)
\end{aligned}
$$

Therefore, $V$ will keep decreasing till $|\omega|=|m|$, which also means that the optimal approximation vectors $\boldsymbol{\theta}_{f}^{*}$ and $\boldsymbol{\theta}_{g}^{*}$ are obtained.

C. Tracking Control

To solve the tracking problem, i.e., the stabilization problem $s=0$, we turn back to the expression (25). In order to force the trajectory to reach the sliding mode $s=0$, we must choose the switching gain to overcome the modelling error $\omega$. We assume that there exists the ideal parameter vector $\boldsymbol{\theta}_{k}^{*}$ such that

$$
\boldsymbol{\theta}_{k}^{* T} \boldsymbol{\xi}(\mathbf{x}) \geq|\omega|+\eta
$$

Then we propose the following adaptive law to adjust the third fuzzy system for approximating the switching gain.

$$
\dot{\boldsymbol{\theta}}_{k}=\gamma_{3} \xi(\mathbf{x})|s|
$$


If we choose the Lyapunov function as

$$
V=\frac{1}{2} S^{2}+\frac{1}{2 \gamma_{3}} \widetilde{\boldsymbol{\theta}}_{k}^{T} \tilde{\boldsymbol{\theta}}_{k}
$$

where $\tilde{\boldsymbol{\theta}}_{k}=\boldsymbol{\theta}_{k}-\boldsymbol{\theta}_{k}^{*}$, we have

$$
\begin{aligned}
\dot{V} & =s \dot{s}+\frac{1}{\gamma_{3}} \widetilde{\boldsymbol{\theta}}_{k}^{T} \dot{\boldsymbol{\theta}}_{k}=\omega s-\boldsymbol{\theta}_{k}^{T} \xi(\mathbf{x})|s|+\frac{1}{\gamma_{3}} \widetilde{\boldsymbol{\theta}}_{k}^{T} \dot{\boldsymbol{\theta}}_{k} \\
& =\omega s-\boldsymbol{\theta}_{k}^{*^{T} T} \xi(\mathbf{x})|s|+\boldsymbol{\theta}_{k}^{* T} \boldsymbol{\xi}(\mathbf{x})|s|-\boldsymbol{\theta}_{k}^{T} \xi(\mathbf{x})|s|+\frac{1}{\gamma_{3}} \widetilde{\boldsymbol{\theta}}_{k}^{T} \dot{\boldsymbol{\theta}}_{k} \\
& =\omega s-\boldsymbol{\theta}_{k}^{*_{T} T} \boldsymbol{\xi}(\mathbf{x})|s|-\widetilde{\boldsymbol{\theta}}_{k}^{T} \xi(\mathbf{x})|s|+\frac{1}{\gamma_{3}} \widetilde{\boldsymbol{\theta}}_{k}^{T} \dot{\boldsymbol{\theta}}_{k} \\
& =\omega s-\boldsymbol{\theta}_{k}^{*_{T} T} \boldsymbol{\xi}(\mathbf{x})|s|+\widetilde{\boldsymbol{\theta}}_{k}^{T}\left(-\boldsymbol{\xi}(\mathbf{x})|s|+\frac{1}{\gamma_{3}} \dot{\boldsymbol{\theta}}_{k}\right) \\
& \leq-\eta|s|
\end{aligned}
$$

In summary, the adaptive laws in (26) lead to the function approximations of fuzzy systems in (16). Meanwhile, with the decreasing modelling error, the adaptive law (30) adjusts the switching gain to the minimum level for overcoming the residual modelling error, therefore unnecessary large magnitude chattering is avoided.

\section{SIMULATIONS}

Given an unstable plant

$$
\dot{x}(t)=\frac{1-e^{-x(t)}}{1+e^{-x(t)}}+u(t)
$$

where $|f(x)|=\left|\frac{1-e^{-x(t)}}{1+e^{-x(t)}}\right| \leq 1$, it is clear that the plant is unstable without control input because if $u(t)=0, \dot{x}>0$ for $x>0$ and $\dot{x}<0$ for $x<0$. To build the proposed approximators, we define six fuzzy sets over the intervals [3,3] with labels $N_{3}, N_{2}, N_{1}, P_{1}, P_{2}, P_{3}$. The Gaussian membership functions are

$$
\begin{gathered}
\mu_{N 3}=\frac{1}{1+\exp (5(x+2))}, \mu_{N 2}=\exp \left(-(x+1.5)^{2}\right) \\
\mu_{N 1}=\exp \left(-(x+0.5)^{2}\right), \mu_{P 1}=\exp \left(-(x-0.5)^{2}\right) \\
\mu_{P 2}=\exp \left(-(x-1.5)^{2}\right), \mu_{P 3}=\frac{1}{1+\exp (-5(x-2))}
\end{gathered}
$$

The control law is

$$
\hat{u}=c\left(x_{d}-x\right)-\hat{f}\left(\mathbf{x} \mid \boldsymbol{\theta}_{f}\right)+\dot{x}_{d}+\hat{k}\left(\mathbf{x} \mid \boldsymbol{\theta}_{k}\right) \operatorname{sign}(x)
$$

where $x_{d}=\sin (t)$ is the desired state trajectory. Here the modeling error is $\omega=\hat{f}\left(\mathbf{x} \mid \boldsymbol{\theta}_{f}\right)-f(\mathbf{x})$ and the tracking error is $e=x_{d}-x$. With adaptive laws (26) and (30), the simulation results are shown in Figures 1-4, which demonstrate the convergence of the modelling error and tracking error is guaranteed with the unknown nonlinear function, and the chattering of traditional sliding mode control is eliminated. This is because the modelling error converges to a very small minimum value rapidly with adaptive law (26) as in Figure 1, therefore the discontinuous gain only needs to be kept at a very small positive value near zero with adaptive law (30), which can still guarantee the convergence of the tracking error as in Figures 2 and 3, and the chattering of control is eliminated as in Figure 4.

\section{CONCLUSIONS}

The proposed modelling-error based adaptive fuzzy sliding mode control scheme guarantees the asymptotic convergence of the modelling error and tracking error. The limitation of conventional tracking-error based adaptive law, which only assures the convergence of tracking error, is released. Furthermore, the bounds of the modelling error are not required a priori. The simulation results validate the usefulness of the proposed controller.

\section{REFERENCES}

1. A. Isidori, Nonlinear Control Systems, 2nd ed. Berlin, Germany: Springer-Verlag, 1989.

2. J. J. E. Slotine and W. Li, Applied Nonlinear Control, Englewood Cliffs, NJ: Prentice-Hall, 1991.

3. V.I. Utkin (1992) Sliding modes in control optimization, Springer-Verlag.

4. C. Edwards and S. K. Spurgeon, Sliding Mode Control-Theory and Applications, London, U.K.: Taylor \& Francis, 1998.

5. L. X. Wang, Adaptive fuzzy systems and control: designing and stability analysis, Prentice-Hall, Englewood Cliffs, NJ, 1994.

6. K. Fischle and D. Schroder, "An improved stable adaptive fuzzy control method," IEEE Transactions on Fuzzy Systems, vol. 7, pp. 27-40, 1999.

7. H. Lee and M. Tomizuka, "Robust adaptive control using a universal approximator for SISO nonlinear systems," IEEE Trans. Fuzzy Syst., vol. 8, pp. 95-106, 2001.

8. Chi-Hsu Wang, Tsung-Chih Lin, Tsu-Tian Lee, HanLeih Liu, "Adaptive hybrid intelligent control for uncertain nonlinear dynamical systems, " IEEE Transactions on Systems, Man and Cybernetics, Part B, vol. 32, no. 5, pp. $583-597,2002$.

9. Jang-Hyun Park, Sam-Jun Seo and Gwi-Tae Park, "Robust adaptive fuzzy controller for nonlinear system using estimation of bounds for approximation errors," Fuzzy Sets and Systems, vol. 133, no. 1, pp. 19-36, 2003

10. J. A. Farrell, "On performance evaluation in on-line approximation for control," IEEE Transactions on Neural Networks, vol. 9, pp. 1001-1007, 1998.

11. J. A. Farrell, "Stability and approximator convergence in nonparametric nonlinear adaptive control," IEEE Transactions on Neural Networks, vol. 9, pp. 10081020, 1998.

12. M. Hojati and S. Gazor, "Hybrid adaptive fuzzy identification and control of nonlinear systems," IEEE Transactions on Fuzzy Systems, vol. 10, no. 2, pp. 198 210, 2002.

13. G. C. Hwang and S. C. Lin, "A stability approach to fuzzy control design for nonlinear systems," Fuzzy Sets Syst., vol. 48, no. 3, pp. 279-287, 1992. 
14. Rainer Palm, Robust control by fuzzy sliding mode, Automatica, vol. 30, no. 9, pp. 1429-1437, 1994.

15. S. W. Kim and J. J. Lee, "Design of fuzzy controller with fuzzy sliding surface," Fuzzy Sets Syst., vol. 71, pp. 359-369, 1995.

16. J.-C. Lo and Y.-H. Kuo, "Decoupled fuzzy sliding mode control," IEEE Trans. Fuzzy Syst., vol. 6, pp. 426-435, 1998.

17. B. Yoo and W. Ham, "Adaptive fuzzy sliding mode control of nonlinear systems," IEEE Transactions on Fuzzy Systems, vol. 6, pp. 315-321, 1998.

18. C. L. Hwang and C. Y. Kuo, "A stable adaptive fuzzy sliding mode control for affine nonlinear systems with application to four-bar linkage systems," IEEE Trans. Fuzzy Syst., vol. 9, pp. 238-252, 2001.

19. F. Da, "Decentralized sliding mode adaptive controller design based on fuzzy neural networks for interconnected uncertain nonlinear systems," IEEE Trans. Neural Networks, vol. 11, pp. 1471-1480, Nov. 2000 .

20. J. Wang, A. B. Rad and P. T. Chan, "Indirect adaptive fuzzy sliding mode control: Part I: fuzzy switching," Fuzzy Sets and Systems, vol. 122, pp. 21-30, 2001.

21. P. T. Chan, A. B. Rad and J. Wang, "Indirect adaptive fuzzy sliding mode control: Part II-Parameter Projection and Supervisory Controller", Fuzzy Sets and Systems, vol. 122 , pp. 31-43, 2001

22. Chih-Min Lin Hsu, C.-F., "Self-learning fuzzy slidingmode control for antilock braking systems," IEEE Transactions on Control Systems Technology, vol. 11, no. 2, pp. 273- 278, 2003.

23. C.W. Tao, M.-L. Chan; T.-T. Lee; "Adaptive fuzzy sliding mode controller for linear systems with mismatched time-varying uncertainties," IEEE Transactions on Systems, Man and Cybernetics, vol. 33, no. 2, pp. $283-294,2003$.

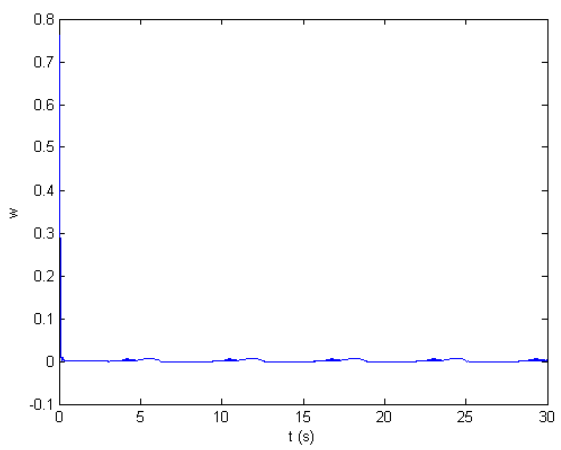

Figure 1: Modelling error

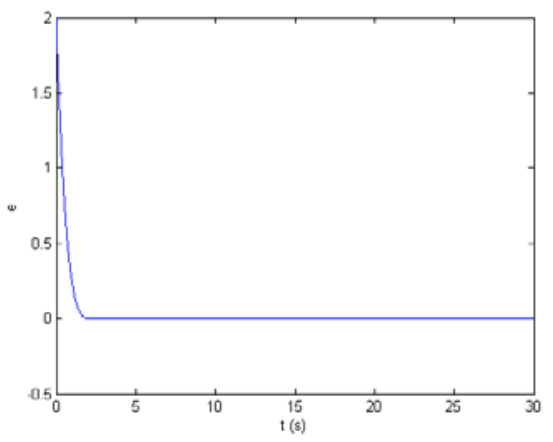

Figure 2: Tracking error

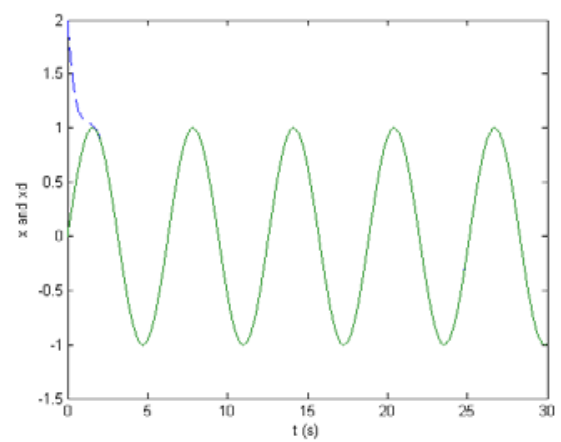

Figure 3: Desired and observed responses

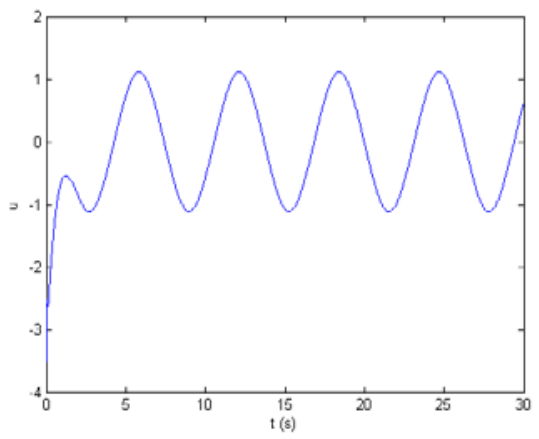

Figure 4: Control signal 